\title{
Register
}

Aberglauben 138, 162

Abhängigkeitserkrankungen 457

Aboriginals 192-202

- Aboriginal-Kinder 193, 195, 197

- Aboriginal-Patienten 193

Aëtios von Amida 115, 117

Affordable care 412

Afrika 126, 158, 162 f., 242 f., 250

Ageing 365

Ahnen 161, 163, 165-167, 206, 211, 215

AIDS 162, 208, 265, 273, 291f., 304, 339, $394,420-429$

- Aids-Hilfe 424

- Aidskörper 420-423, $428 \mathrm{f}$.

- Aids-Krise 425, 431

- Aidspolitik 423, 427

Aktuarios, Johannes 116, 126

Akupunktur 158, 161

Akutbehandlung 458

- Akutfall 482

ALARA-Prinzip $381 \mathrm{f}$.

Alarmin 267

Alexandros von Tralleis

Alkohol 118, 161, 458

- Alkoholabhängigkeit 458

- Alkoholentzug 458

Allergien 266, 274, 276, 441, 478, $481 \mathrm{f}$.

Allgemeingefährdung 425

Allgemeinmedizin 75, 205

Allgemeinversorgung 157

Allgemeinwohl 378

Alter 35f., 135, 180, 248, 250 f., 326, 456

- Altenheime 122

- Alterserscheinungen 250

- Alterssicherung 355

- Altersvorsorge 31

Amazonas 170-183, 308

- Amazonas-Kulturen 174

- Amazonas-Medizin 177, 180

Amerika 57, 172f., 261, 317f., 367, 392 394, 412, 444

Amputation $83 \mathrm{f} ., 86 \mathrm{f}$.

Amulette 114, $119 \mathrm{f}$.
Anabolika 65

Anamnese 72,75

Anästhesie 177

Anatomie 19, 151, 223, $227 \mathrm{f.,} \mathrm{237,} \mathrm{251,} 294$

Andronikos I. Komnēnos 123

Angemessenheit. Siehe Appropriate Care

Angst 46, 127, 162, 248, 295f., 314, 328, $337,344,421,474,490$

- Angststörung 180

Anschlussheilbehandlung 487

Ansteckung 173

- Ansteckungsgefahr 19, 21

- Ansteckungsrisiko 377

Anthemios 118

Anthropologie 44, 48, 50 f., 80, 355 f., 452

- Anthropomorphismus 22

- Anthropotechnik 64,66f.

Anthropozentrismus 296

Antibiotika $214,253,261,263,268,272$ f., $276,436,441$

- Antibiotikaeinsatz 300, 306

- Antibiotikaforschung 272

- Antibiotikageneration 238

- Antibiotikamissbrauch 279

- Antibiotikaresistenz 249, 281, 446

- Antibiotikaverbrauch $272 \mathrm{f}$.

Antidepressiva $\quad 65,178$

Antidotarium 126

Antigen 271

Antiinfektiva $272 \mathrm{f} ., 436$

Antike 2f., 8 f., 15 f., 18, 30, 32-39, 44, $70-81,82-92,98,104$ f., 109 f., $113-$ $118,126,128-130,142,171,401$

Antikörper 265, 273f., $479 \mathrm{f}$.

Anti-Projektions-Mechanismen 25

Antitoxine 231

Antonovsky, Aaron $\quad 26,158,312,316$

Antyllos 117

Apartheid $159 \mathrm{f}$.

Apollon 85

Apotheke 20, 158

Apparatemedizin 357

Appropriate Care 401-403, 405, 409 f., 416

Ә OpenAccess. (c) 2021, publiziert von De Gruyter. (cc))BY-NC-SA Dieses Werk ist lizenziert unter einer Creative Commons Namensnennung - Nicht kommerziell - Weitergabe unter gleichen Bedingungen 4.0 International Lizenz. https://doi.org/10.1515/9783110713336-042 
Araber 115, 120-122, 125, 129

Arbeit 75, 120, 323, 340-347, 479

- Arbeitgeber 346, 413, 495

- Arbeitsbelastung 340-344

- Arbeitslosenversicherung 365, 368

- Arbeitslosigkeit 159, 161, 358, 380

- Arbeitsmigration 159

- Arbeitsschutzregelungen 346, 355

- Arbeitsunfall 91

- Arbeitszufriedenheit 346

- ArbZG 346

Archäologie 172, 248

Aristoteles 17, 32, 37-40, 73, 126, 401, 416 Armenien 128

Arten $34,175,178,208,212,242,249,305$, 308

- Artensprung 261

- Artensterben 306

- Artenvielfalt $175,177 \mathrm{f}$.

- Artgerechtheit 294

- Artgrenzen 246

Arznei $16,78,120,125,130,149,151,250$, $390 \mathrm{f.,} 478$

- Arzneimittelmarkt 357

- Arzneimittelstudien 358

- Arzneivorschriften 126

- Arzneiwirkstoffe 357

Arzt $16-20,23,32-36,50 f ., 62-67$, $71-79,83-87,98$ f., 104, 110 f., $113-$ 120, 123-127, 146, 339-347, $404 \mathrm{f}$., $408 f ., 412 f ., 415,436,439 f$.

- Arztbrief 342, 483

- Ärztebudget 481

- Ärztemangel 341

- Arztgesundheit 340, 343, 345, 347

- Arzt-Patient-Verhältnis 63,67

- Arzt-Patient-Vertrauensverhältnis 66

Arzt-Gelehrter 125

Arzt-Heiliger 83, 85

Asien 158, 431

Asklepios 30, 33, 111, 114

Asklepiosheiligtümer 85,122

Atem 136f., 206f., 265, 324, 388

- Atemseele 206, 208

- Atemstörungen 302

- Atemwegserkrankungen 214

Athanasius 111
Aufklärung $91,208,280,307,373,441$, 443, 445, 485

Aufklärung (Epoche) 65, 244, 299, 355

Aufmerksamkeitsdefizit $\quad 58,345$

Aufmerksamkeitsökonomie 388

Augen $7,52,56,63,86,103,118,138,180$, 263, 272, 302, 304, 374, 484

- Augenarzt 227

- Augenkrankheiten 119

- Augenwurm 305

Augustinus $19,43,111$

Ausbildung 9,39f., 91, 116, 124, 148, 160 f., 167, 227, 410, 439, 473, 492, $494 \mathrm{f}$.

Ausbreitung 263, 265, 367, 435

- Ausbreitungseffektivität 292

- Ausbreitungsgeschwindigkeit 263, 377

- Ausbreitungswege 377

Ausgangsbeschränkungen 305

Aushandlung 161, 402, 408, 415

Aussatz 115, 261

Australien 173, 192 f., 195-199, 201, 363, $367-371,440-442$

Austreibung 115

Autismus 254

Autoimmunkrankheit 243, 266, 479

Automatismen 62

Ayahuasca 178, 180 f., 278

- Ayahuasca-Tourismus 180

Āyurveda 146-154, 177

- Ayurveda-Experten 148

- Ayurveda-Resort 148

Bacon, Francis 56

Bakterien 26f., 138, 240, 246 f., 249, 263 f., $268,273,276-278,435$

Bakteriologie 26f., 138, 228, 230

Bakteriophagen 273

Bauch 135, 478

- Bauchbeschwerden 481

- Bauchfellentzündungen 436

- Bauchschmerzen 160

Bauern 83, 86f., 91, 428

- Bauernverband 294

Baumgartner, Isidor 90

Bedürftigkeit $122,124,280$ 
Befunden 224, 227, 312, 315f., 326, 333, $342,366,408,427,454-456$

Behandlung 9, 17, 33-35, $37 \mathrm{f} ., 50,52,58$, $60,76,90,119,123 \mathrm{f} ., 148,152,165$, $178-180,182,193,207,211,215,249$, $273 \mathrm{f} ., 276,315,326,334,339,343 \mathrm{f}$., 358, 363f., 405-407, 410, 415, $435 \mathrm{f}$., 438, 441-443, 458, 469

- Behandlungsabbrüche 458

- Behandlungsbedürftigkeit 343

- Behandlungsfehler 365

- Behandlungshoheit 439

- Behandlungsnotwendigkeit 445

- Behandlungsqualität 404

- Behandlungszeit 165

Behinderte 2, 33, 36, 138, 317-319, 327, 468, 472f., 482

- Behindertengrad 482

- Behindertenstatus 482

Behring, Emil von 227, 230 f., 261, 436

Beischlaf 121

Belastungsstörungen 366

Beruf $24,111,161,202,223,323,336$, $339-341,343,346 \mathrm{f} ., 471$

- Berufskrankheiten 345

- Berufszufriedenheit 343

Beschneidungsgebot 102

Beschwerden 9, 36, 75, 207, 210, 248, 314, 319, 358, 458, 478, 480f., 495

Beschwörungsformeln 179

Besprechungsformeln 114

Best-Practice-Beispiel 369

Beulenpest $19,126,421$

Bevölkerung $79,135,141,147,172-174$, 180 f., 196f., 228, 245, 250 f., 354, 357, 365, 374, 388, 412f., 415, 426, 436, $438 \mathrm{f} ., 443,445$

- Bevölkerungsdichte 173, 261

- Bevölkerungsschutz 262

- Bevölkerungszunahme 244

Bewegung 9, 33, 75, 103, 113, 118, 137, 192, $199-202,253,298,380,470$ f., 476, 489

- Bewegungsförderung 192, 198

- Bewegungskindertagesstätte 202

- Bewegungskultur 192-194, 198, 201203
- Bewegungsmangel 196, 245

- Bewegungsspiele 193

- Bewegungswissenschaft 52

Bibel 98, 103

Bilder 86, 91, 210, 314, 420 f., 427

- Bildgebung 472

- Bildsammlung 112, 129

- Bildspender 19

- Bildsprache 27, 423, 428

Bildung 47, 159, 193, 197, 202, 224, $243 \mathrm{f}$, 253, 280, 326, 330, 332f., 369, 486

- Bildungspolitik 194

- Bildungsreform 222

- Bildungssystem 39

Blut $8,16,113,121,127,140,152,206$, 213f., 223, 266, 269, 324, 470f., 476, 479 f., 488

- Blutanalysen 224

- Blutgerinnung 250

- Bluthochdruck $\quad 6,36,140,315,326,468$, 470, 477, 488

- Blutkreislauf 23, 109

- Blutschuld 103

- Blutvergiftung $\quad 83,88,90,435,441$

- Blutzuckerspiegel 470

Breuer, Josef 331

Brust 243, $315 \mathrm{f} ., 486$

- Brustkorb 472

- Brustkrebs 486

Budget 391f., 481

- Budgetkürzungen 392

Bundesgesundheitsminister $\quad 440$

Bürokratisierung 341, 345

Byzanz 85, $110 \mathrm{f} ., 113-116,118,120-126$, 128,130

Canguilhem, Georges 25

Cartwright, Nancy 27, 59

CAR-T-Zell-Therapien 233

Cellularpathologie 225f., 239

Chemotherapie 302, $486 \mathrm{f}$.

China 30, 133-136, 138f., 141f., 174, 305, $387 \mathrm{f} ., 392-394$

Chirurg 17, 116, 228f., 313

- Chirurgenschule 227

- Chirurgie 15, 17, 19, 35, 65 f., 110, 113, $121,227-229$ 
Christentum 15, $18 \mathrm{f} ., 27,82 \mathrm{f} ., 85 \mathrm{f} ., 89 \mathrm{f} .$, 92, 99, 102, 109-111, 113f., 122, 162, 206, 209, 215

Cicero 18

Computer $\quad 60,325,329-331,334,416$

- Computerspiele 245

COVID-19 4, 10, 27, 39, 41, 59, 61, 80, 105f., 141-143, 182, 197, 238, 253, 262 f., 270 f., 273-275, 280, 291, 303, 305, 339f., 367, 374, 376-378, $380 f .$, 387-389, 392f., 397, 406, 434 f., 437, $447 \mathrm{f} ., 483,491$

Daoismus $134 \mathrm{f} ., 137$

Darm 118f., 276f., 481

- Darmbakterien 277

- Darmerkrankungen 277

- Darmkrebs 315

- Darmspiegelung 481

Darwin, Charles 236, 244 f., 250

Daten $31,35,59-64,67,141,147,226$, $237-239,268,330,335,340,363-374$, $376,378,411,414-416,492$

- Datendiebstahl 368

- Datenerfassung 61

- Datennutzung 364, 368, 370

- Datenschutz 368, 370, 372

Defektzuchten 307

Deleuze, Gilles 72

Demenz 2, 180, 324-327, 332f., 336, $456 \mathrm{f} ., 460 \mathrm{f}$.

Demokrit 16

Depression 37, 57f., 178, 180, 248, 253, 278, 324, 326, 339, $344 \mathrm{f.,} \mathrm{451,} \mathrm{461,} 480$

Derrida, Jacques 20, 25

Descartes, René 20

Desinformation 280, 307

De Souza, Jonas Gregorio $\quad$ 172-174

Deutung $19,57,72,212,357$

- Deutungshoheit $56 \mathrm{f}$.

- Deutungsmonopol 102

Diabetes $31,36,40,52,140,160,196,245$, $268,277,326,438$

Diagnose $2,9,22-25,37,57 \mathrm{f} ., 67,116$, $125,142,148,180,207,209$ f., 215, 224, 226, 233, 238f., 250, 253f., 268, $270-$ $274,324,327,332,343,363,365,402$, 405f., 441, 447, 455f., 468, 470, 472, 474, 479-481, 487, 490

- Diagnosekriterien 451

- Diagnoseplattform $270 \mathrm{f}$.

Diät $\quad 35,72,359$

Diätetik 35f., 99, $104 \mathrm{f.,} \mathrm{113-115}$

Dienstleistungsmedizin 65

Digitalisierung 64,364

Digitalpakt 202

Diokles von Karystos 32, 35

Dioskurides 110,117, $129 \mathrm{f}$.

Diphtherie 230f., $261 \mathrm{f}$.

Diskriminierung $61,197,368,409,423$, 425, 429, 431, 484

Disziplinargesellschaft 19

DNA 31, 237, 240-242, 246

- DNA-Einzelstrangbrüche 246

- DNA-Impfstoffe 271

- DNA-Reparaturmechanismen 246

Domestikation 174, 261, $294 \mathrm{f}$.

Doșa 150-153

- Doșa-Konstellation 151

- Doșa-Lehre 150

Dosierung 232, 270

Dosis-Wirkungs-Kurve $378 \mathrm{f}$

Droge 197, 366, 422, $458 \mathrm{f}$.

- Drogenabhängige $459 \mathrm{f}$.

Dwārakānātha, Chandragiri 147, 150, 152

Ebola 253, 274, 291f., 392, 394-396, 435

Effektivität $\quad 40,406,444$

Effizienz $61,300 \mathrm{f} ., 343,346,408$

e-Health 236

Eingeweidewürmer 119

Einkommen 270, 380, 435

Einwanderung 26,173

Einzelzellanalyse $232 \mathrm{f}$, 269

Elenktik 77

Emotionen 58, 88 f., 163, 193, 296, 329

Empathie 344, 451, $453 \mathrm{f}$.

Empfindlichkeit 33

Empfindungsfähigkeit 297

Empirie $20 \mathrm{f.,} \mathrm{35,} \mathrm{55,} \mathrm{59,} \mathrm{223,} \mathrm{335,} \mathrm{366,}$ 403, 406, 414, 454

Enhancement $\quad 64-66,402,405$

Entgiftung 458

Entkolonialisierung 394 
Entscheidungsprozesse $\quad 391,407,440,447$, 462

Entwicklungsländer 253

- Entwicklungspolitik 395

Entwicklungsstörung 238, 249

- Entwicklungsfelddefekte 249

Entzug $458 \mathrm{f}$.

Entzündung $90,274,277$ f., 300, 302

Enzym 178, 250, 274

Epidemie 8, 34, 36, 126, $138 \mathrm{f} ., 173,181 \mathrm{f}$., 261-263, 280, 291, $425 \mathrm{f} ., 431,436$

Epigenetik 241, 252

Epikureer 17, 24

Erbgut 239f., 243-246, 248, 263, 265, $269-271$

Erkrankung 2, 31, 33-36, 39, 72, 79, 86, 127, 149, 160, 196, 202, 207-210, 212, $237,239,247,253,277,317-319,323$, $333,336,344,366,438,442,450-453$, $455-459,461$ f., 468 f., 472, 479-483, $490 \mathrm{f} ., 495 \mathrm{f}$.

- Erkrankungsrisiko 316

Erlebniswelt 56, 62, 67

Ernährung $33,103,120,136,148,151$, 195f., 248, 253, 280, 295, 299, 301, 468, 470, 476

- Ernährungsbedingungen 244

- Ernährungsberatung 148

- Ernährungsdiätetik 114

- Ernährungsgewohnheiten 301

- Ernährungstherapeutin 487

Erreger 227, 248, 265f., 268, 271, 273f., 291f., 304, 378, 380, 435, 455

- Erregerdiagnostik 446

- Erregerverbreitung 292

Erschöpfung 127, 340, 344, 487

- Erschöpfungsdepression 344

- Erschöpfungssymptomatik 344

Erwerbsminderungsrente 479

Essstörungen 253

Ethik $16-18,20$ f., 37, 51, 54, 146, 297, 299, 346, 353, 355f., 372, 401, 404, 407, $415 \mathrm{f}$.

Ethnozentrismus 25

Eugenik 252

Eukrasie 113

Eunapios von Sardeis 117
Eurozentrismus 171

Eusebius 111

Evolution $7 f$., 109, 239-241, 244-246, 249, 251f., 292, 296f., 452

Experimentalchemie 228

Experimentalphysik 228

Experimentalphysiologie 223

Experimentaltechniken 178

Experten 33, 52, 133, 141-143, 174, 233, 369, 383, 388 f., 402, 405, 410, 426, 441, 462, 481

Facharzt $\quad 478,480,488$

- Facharztanerkennung 439

Fadenwürmer 260

Fall-Kontroll-Studien 370

Fehlernährung 295

Fehlhaltungen 340

Fehltage $472 \mathrm{f}$.

Fehlversorgung 402, 409

Fieber $8,115,119,248,278,435,447$

Finanzierung $41,59,160,209,226,238$, 300 f., 323, 343, 359, 364, 377, 383, 391, 397, 402, 406 f., 409 f., 412, 414 f., 435, 442, $446 \mathrm{f} ., 483,491$

Finanzkrise 394

Fitness $35 \mathrm{f} ., 60,198,248,303,308,429$, 476

- Fitnessarmbänder 54

- Fitnessstudio 476

Fledermäuse 274, 292

Fleisch 152, 295f., 301, 306 f., 470

- Fleischhandel 294

- Fleischhygiene 308

- Fleischkonsum 275

Flow-Cytometry 268

Flugverkehr 263

Fodor, Jeremy A. $329 \mathrm{f}$.

Forschung $3,25,32,34,40,67,83,143$, 149, 172, 181, 183, 222, 226, 229, $232 \mathrm{f}$. , 237 f., 253, 267 f., 272 f., 275, 280 f., 292, 324, 353 f., 363f., 368, 370, 372f., 393, 397, 405, 421, 436, 438, 440, 490, 492

- Forscherpersönlichkeiten 436

- Forschungsbedarf 254

- Forschungsdatenzentren 368

- Forschungsdefizite 307 
Forstwirtschaft 175

Forsyth, Miranda 213

Foucault, Michel 19, 89, 359

Fragmentierung 9,59

Framingham-Studie $313,315 \mathrm{f}$.

Freizeit 193, 197, 302, 326, 341, 346

Freud, Sigmund $\quad 3,25,46,84,87 \mathrm{f} ., 98,119$, 137, 141, 296, 303, 331f., 347, 461, 476, 480, 484

Freund-Feind-Schema 88

Fromm, Erich 49, 128

Früherkennung 238, 314f., 319, 440, 445

Frühwarnsysteme 367,446

Fukuyama, Francis 354

Gadamer, Hans-Georg $\quad 3,43,55,141,312$

Galen 32, 36, 87 f., 105, 109 f., 113 f., 117 f., $124 \mathrm{f} ., 130$

Ganzheitlichkeit 7 f., 40, 48, 50, 80, 83, 91f., 102, 140, 166, 177, 202, 237, 249, 253 f., 295, 454, 491

Ganzheitsarzt 478

Geburt 32, 77, 91, 120, 139, 151, 206, $213 \mathrm{f}$., 244, 251, 364, 367, 370

Gedächtniszelle 270

Gefängnis 458,461

Gefühl 2, 22, 46 f., 49, 57, 140, 162, 166, 208, 212, 278, 294, 312-319, 342, 451, 476

- Gefühlsantrieb 46

- Gefühlsleben 326

- Gefühlsverfassung 211

Gehirn 16, 120, 180 f., 243, 245, 251, 264 , $277,296,324-327,329-331,333 \mathrm{f}$.

- Gehirnbiologie 328

- Gehirnentzündung 292

- Gehirnkrankheit 455

- Gehirnregionen 328

- Gehirnzellen 181

Gemeinschaft $18 f ., 70,166,180,182,196$, 198, 203, 212, 216, 280f., 353, 356, 358f., 406, 431, 468, 474, 493

Gemeinwohl $\quad 40,355,359,382,447$

Genesung $50,64,72,211,336,468,487$

Genetik 79f., 236, 238f., 241f., 246f., 252, 271, 294, 300, 328

- Genmutation 245
- genomDE 239

- Genommedizin 239

- Genschere 405

Genozid 27, 195

Gerechtigkeit 401

Geschlechterbeziehungen 212

Geschlechtskrankheiten 118, 421

Gesetz 84, 102, 106, 124, 159, 193, 299, 307, 358, 368, 438, 446, 491

Gesprächstherapie $\quad 454,473$

Gesundheitsamt 135, 227

Gesundheitsarmbänder 60

Gesundheitsausgaben $\quad 402,408$

Gesundheitsbegriff $4-6,32,37,43,45,49$, 64, 79 f., 82, 86, 91f., 98, 114, 140, 154, 177, 192, 194, 202, 205, 312, 315, 322f., 331, 333, 336, 454

Gesundheitsberichterstattung $79,364,367$, 441

Gesundheitsberufe $\quad 9,209,346,412,415$

Gesundheitsbildung 140

Gesundheitsdaten 61, 238, 363-366, $368 \mathrm{f} ., 373 \mathrm{f}$.

Gesundheitsdienstleistungen $\quad 80,213 \mathrm{f}$., 216, 346, 364, 436, $439 \mathrm{f} ., 446 \mathrm{f}$.

Gesundheitsdiktatur 61, 359

Gesundheitsförderung 317, 319

Gesundheitsforschung 365

Gesundheitsfürsorge 105,135

Gesundheitsgefährdung 56

Gesundheitsgefühl 314f., $318 \mathrm{f}$.

Gesundheitsgerechtigkeit 202

Gesundheitsgesetzgebung 102

Gesundheitsgewinn 319

Gesundheitsindikatoren 365

Gesundheitskompetenz 140

Gesundheitskrise 262

Gesundheitsminister 160, 371f., 377, 391, 396, 426, 441

Gesundheitsniveau 389

Gesundheitsökonomie 60

Gesundheitspflege 135

Gesundheitspolitik 6f., 10, 31, 40, 139, 358, 393, 397

Gesundheitspotenzial $40 \mathrm{f}$.

Gesundheitsprobleme 31, 263, 300, 303, 393 
Gesundheitsregeln 104

Gesundheitsrisiken 305f., 340, 367

Gesundheitsservice 193

Gesundheitssicherheit 392

Gesundheitsstadt 5

Gesundheitssystem 9f., 31, 39f., 79, 193, 202, 341, 343, 363, 368, 384, 406, 408-410, 429, 435f., 438, 442, 444, 469, 483, 495

Gesundheitstheorie 336

Gesundheitstourismus 182

Gesundheitsvergessenheit 43

Gesundheitsverhalten $365 \mathrm{f}$.

Gesundheitsversorgung $6 \mathrm{f} .$, 9, 39f., 157, 159 f., 180, 214 f., 232, 239, 355, 393f., 402, 405, 408, 410, $415 \mathrm{f}$.

Gesundheitsvorschriften 262f., 394-396

Gesundheitsvorsorge 35, 102

Gesundheitswahn 52

Gesundheitswesen $\quad 6,65,79,134,159,238$, $273,280,340-342,345-347,370,391$, 401-403, 405 f., 408-412, 415 f., 436, 438, 440, 442, 445, 447f., 490, $494 \mathrm{f}$.

Gesundheitswirklichkeiten 64

Gesundheitswissenschaft 70, 75, 79f., 313, 317

Gewebe 71, 73, 75, 151-153, 241

- Gewebearten $150 \mathrm{f}$.

- Gewebelehre 225

- Gewebeproben 327, 481

Gewinnmaximierung 408

Ghebreyesus, Tedros Adhanom 393

Glaube $65,111,113,128,162,165,178,215$, 245,323

Glücksforschung 2, 480

Grippe $173,253,261,265,291,435,441$

Grundlagenforschung 252,268 f., 278, $298 \mathrm{f}$.

Guattari, Félix 72

Habermas, Jürgen 25

Handlung 17, 38, 51, 57, 63f., 84, $87 \mathrm{f}$, $103 \mathrm{f} ., 116,140,143,161,165,299 \mathrm{f} .$, 376 f., 381, 425, 451

- Handlungsaufforderung 61

- Handlungsimpulse 460

- Handlungskaskade 63
- Handlungspflicht 61

Harnack, Alfred von 18

Harré, Rom 332

Hausarzt 222, $470 \mathrm{f}$.

Haustiere 292f., 302 f., 306

Hebammenwissenschaft $52,77,214$

Heidegger, Martin 48, 63

Heiler 18f., 114, 124, 158, 160-167, 176, $179 \mathrm{f.,} \mathrm{182,} \mathrm{193,} \mathrm{207,} 215$

- Heiler-Ausbildung 160

- Heiler-Organisationen 160

- Heiler-Traditionen 163, 166

- Heilriten 180

- Heilrituale 114

- Heilzeremonie 179

Heiligenverehrung 113

Heilkunde $15 \mathrm{f} ., \mathrm{73}, 98,109,114,125,128$, 140, 147, 159

- Heilerfolge 86

- Heilerlebens 163

- Heilkräfte 125

Heilung 17-21, 24f., 34, 64, 70 f., 83, $85-88,90,111 \mathrm{f} ., 114,140,142,158$, 163, 165-167, 182, 192f., 207, 209, 211, 233, 274, 318, 336

- Heilkuren 118

- Heilmittel 35, 114, 118f., 230, 292, 404

- Heilpflanzen $158 \mathrm{f}$.

- Heilpraktiken 119, 159, 180, 193

- Heilprozess 142, 166

- Heiltätigkeit 114

- Heilungsmessen 215

- Heilungsmodell 182

Heliodoros 117

Herdenimmunität 365

Hermeneutik 60-62

Herz 16, 61, 88, 103, 137, 245, 264, 313, $315,326,333,415,438,443,470$

- Herzfehler 327

- Herzinfarkt 442f., 445

- Herz-Kreislauf-Erkrankungen 246, 277

- Herz-Kreislauf-System 326

Hesiod 70

Heuristik 22, 355

Hexerei 162, 167, 208

Hierarchie 18, 346

High-Tech-Medizin 358 
Hippokrates $8,16,32-34,36,71-76,78$, 80, 105, 109 f., 113, $124 \mathrm{f.,} 305$

Hirn-Computer-Schnittstellen 405

Hirnfunktionen 329

Hirnhautentzündung 304

Hirnpathologie 332

Hirnregionen 329

Hirntraumata 333

Hirnverletzungen 327

Hirst, Martin 161

HIV. Siehe AIDS

Hobbes, Thomas 353, 355, 359

Hochdurchsatzmethoden $238 \mathrm{f}$.

Holismus. Siehe Ganzheitlichkeit

Holocaust $26 \mathrm{f}$.

Homer 16, 32, 70

Homöostase 151, 237, 246

Homosexualität $\quad 425,459$

Humangenomprojekt 239

Humorallehre 105, 109, 113

Hunde 160, 295, 298, 302-305, 307

- Hundegeburtstag 302

- Hundegesundheit 307

- Hundeimporte 305

- Hundekuchen 302

- Hunderasse 295, 298, 302

Hunger 46, $104 \mathrm{f.,} \mathrm{128,} \mathrm{158,} \mathrm{295,} 435$

Husserl, Edmund 56

Hygiene 21, 24-26, 99 f., 102, 135, 138, 154, 227, 280, $304 \mathrm{f.,} \mathrm{314,} \mathrm{431,} \mathrm{439,} 446$

- Hygiene-Ausstellung 99

- Hygiene-Bewegung 138

- Hygiene-Maßnahmen 138

- Hygienevorschriften 99

Hyperhagios 111

Hyperspezialisierung 9

Ideenlehre 16

Immunsystem $\quad 8,20,99,152,227,230,232$, 241, 243, 264-271, 274, 276-280, 421, 429, 435 f., 479

- Immunisierung 270

- Immunmodulation 268

- Immunpathologie 266, 274, 276

Impfung 214, 261f., 268-272, 280, 365, 382, 390, 393, 397, 436, 441

- Impfdiplomatie 393
- Impfgegner 280

- Impfnationalismus 393, 397

- Impfpflicht 41

- Impfplattform 270

- Impfquote 365

Indikation 229, $404 \mathrm{f} .$, 407, 414

Infekt $8,80,115,173,238,244,246,253$, $260-263,268-273,276$ f., 279 f., 291, 304 f., 365, 382, 427, 429, 435-439, 445-447, 472, 486

- Infektanfälligkeit 468, 472

- Infektionsbiologie 260f., 268, $279 \mathrm{f}$.

- Infektionsmedizin $439 \mathrm{f}$., $446 \mathrm{f}$.

- Infektionsprävention 436, 446

- Infektionsquelle 305

- Infektionsrisiko $379 \mathrm{f}$.

- Infektionsschutz 380, 446

Interessengruppen $369,373,446$

Introjektionsparadigma 50

IT-Infrastruktur 238

Jasper, Karl 22, 25, 55, 323, 328, 336, 453

Jesus Christus $18 \mathrm{f} ., 110 \mathrm{f} ., 114 \mathrm{f}$.

Judentum 97-99,102, $105 \mathrm{f}$.

Jüdische Speisegesetze 99, 102, 106

Justinian $86,115,117,126,128$

Kanalisation 152, 228, 280

Kant, Immanuel 20-25, 51

Kardinalsäfte 113

Karies 453

Kaschrut-Zertifikat 106

Kassenpatienten 409

Katze 298, 302f., 305

- Katzenkrebs 302

Kauterisierung 123

Kind 31, 35, 58, 193f., 197-202, 214 , 230 f., 304, 366, 369, 445, 470

- Kindbettfieber 261

- Kinderärzte 304

- Kindererziehung 36

- Kindergesundheit 202

- Kinderheilkunde 228

- Kinderkrankheiten 265

- Kindersterblichkeit 197

Kinetik 150

Kirchenvater 43, 105 
Klima $\quad 35,75$ f., 80, 148, 276, 389

- Klimagesundheit 79

- Klimawandel 79, 173, 212, 249, 253, 275, 279, 292, 306

Kloster 123, 130

- Klostermedizin 19

Koch, Robert $\quad 26,201,227$ f., 230, 269, 304, 340, 365, 436, 440 f., 447, 470, 490

Kohärenzgefühl 312,316

Kohlenhydrate 295, 470

Kohortenstudien 370

Komorbiditäten 268, 277

Konsumverhalten 183, 308

Körper 7-9, 16, 18f., 23, 26, 32-35, 37, 44, 46, $48 \mathrm{f}$., 55, 59f., 62, 71f., $77 \mathrm{f}$., 83 f., 87-89, 98, 102-104, 113f., 124, 127, 136, 151f., 165, 192, 200 f., $206-$ 208, 211-214, 223, 247f., 264-266, 269, 271, 273, 276f., 313-319, 355, 359, 420, 427-429, 431, 435, 471, 473, 476, 478, 482, 494

- Körperbau 152

- Körperflüssigkeiten 152

- Körperfreudigkeit 90

- Körpergefühl 489

- Körperhass 90

- Körperhöhlen 121

- Körperpflege 35

- Körpersäfte 16f., 90,113

- Körpersprache 23

- Körperteile 9, 16, 141

Koselleck, Reinhart $\quad 88,90$

Kosmas 83, 90

Kosmetika 299

Kosmos 8, 70 f., 166

Krankenberichte 231

Krankenhaus $122 \mathrm{f} ., 130,135,146,228,231$, 233, 346, 412f., 435, 442, 445, 471f., 474

Krankenhaushygiene 279

Krankenhaussterblichkeit 441-443

Krankenkasse $36,40,368,404,440,458$, 482, 484, 491

Krankenkassendaten 238

Krankenschwester 279, 490

Krankenstation 135

Krankensterblichkeit 444
Krankenversicherung $31,355,458$

Krankheitsbegriff $5 \mathrm{f} ., 22,25-27,44,57$, 59, 63 f., 66 f., 114, 158, 206, 209, 211, $215,292,325,328,366,438,457,462$, 478, 485

Krankheits-Dämonen 98, 115

Krankheitsdiathesen 151

Krankheitsepisode 209

Krankheitserfahrung $\quad 63,67,79,344,468$

Krankheitserreger 173, 264-266, 269 275, 291, 308

Krankheitsfeststellung 63

Krankheitsgeschichte 86

Krankheitskataloge 462

Krankheitslehre 105, 228

Krankheitsmetaphern 19, 26

Krankheitsneigungen 300, 303

Krankheitsprophylaxe 99

Krankheitsrisiko 314

Krankheitsspektrum 115, 292

Krankheitsstadien 142

Krankheitsstatus $457 \mathrm{f}$.

Krankheitsstreben 50

Krankheitssymptome $\quad 20,151$

Krankheitsursachen 208, 237, 247

Krankheitsveranlagungen 302

Krankheitsverhinderung 319

Krankheitsverläufe 224

Krankheitsvorbeugung 31

Krankheitswert 57

Krankheitswirklichkeit 62

Krankheitszustand 63

Kränklichkeit 18

Krankschreibung 487

Kräuter/Langlebigkeits-Medizin 133

Kräutermedizin 215

Krebserkrankung 6,31, 102, 158, 238, 243, 264 f., 269, 274, 299, 314 f., 317-319, 366, 421, 438, 467, 472, $486 \mathrm{f}$., 490, 493

- Krebs-Früherkennung 315

- Krebshärtefonds 487

Kriegschirurgie 121

Kriegsrhetorik 27

Krise 2, 39, 59, 62, 72, 106, 163, 181, 377, 380, 384, 389, 394-396, 434, 448

- Krisenkommunikation 280 
- Krisenmanagement 308

Kruger, Daniel J. 159 f., 251, 253

Kultur 23, 27, 44, 63, 109, 122, 143, 157, 171 f., 174 f., 192-196, 202, 205 f., 244 f., 421, $452 \mathrm{f}$.

- Kulturfähigkeit $243 \mathrm{f}$.

- Kulturgüter 198

- Kulturkreis 125, 142, 176

- Kulturlandschaft 172

- Kulturleistungen 183

- Kulturpessimisten 421

- Kulturraum 122

Kuren 119

- Kurklinik 484

\section{Laienmedizin 90}

Landwirtschaft $\quad 86,174$ f., 272, 299, 306

Langzeitüberlebende $318 \mathrm{f}$.

Langzeitwirkung 214, 366

Leben 2, 7-9, 23f., 26, 31, 33-38, 40 f., $45 f ., 48,50-52,56,61-63,70$ f., 73 , 75 f., 80, 83-85, 87 f., 91f., 97, 99, $103-$ $106,109,113,115,117,121,126,128$, 133-136, 139-141, 149, 151-154, 161163, 167, 172, 176, 182, $192-194,196 \mathrm{f}$., 201, 206, 223, 225-227, 232f., 239241, 245 f., 248 f., 251f., 263, 268 f., 280 , 293, 300, 308, 313, $315 \mathrm{f} ., 319,322 \mathrm{f}$. , 327, 331, 335, 339 f., 354 f., 358, 364, $366,380,384,425,428 f ., 431,438$, 451, 454, $457 \mathrm{f}$., $460 \mathrm{f} ., 468 \mathrm{f}$.

- Lebensalter 113, 151, 490

- Lebensentwürfe 55

- Lebenserfahrung 45

- Lebenserinnerungen 223

- Lebensform 89, 240

- Lebensfreude 312, 489

- Lebensgefahr 106

- Lebensgemeinschaft 166, 295

- Lebensgewohnheiten 63

- Lebensglück 315

- Lebensgrundlagen 86

- Lebenskraft 20, 25, 136 f., 223, 225

- Lebenskunst $51 \mathrm{f}$.

- Lebenslust 429

- Lebensphilosophie 166

- Lebenspneuma 121
- Lebensqualität $31,33,36,65,306,405$, 429, 468, 491

- Lebensraum 179, 276

- Lebensrecht 176

- Lebenssituation 120, 160, 407

- Lebensspanne 135, 266

- Lebensstandard 336

- Lebensstil 38, 40, 79f., 136, 196, 252, 254, 468

- Lebensstilberatung 40

- Lebensstilmedizin 9,40

- Lebenswandel $86 \mathrm{f}$., 431

- Lebensweise 75f., 154, 196, 306

- Lebenswille 50

- Lebenswirklichkeit $59 \mathrm{f}$.

- Lebenswissen 56

Lebensmittel $103,106,136,196,200,266$, 301, 306, 481, 484

- Lebensmittelallergien 483

Leiblichkeit $16,18,23-25,32,43-52,62$, 90, 97 f., 102-104, 143, 182, 354, 358

- Leibarzt 116f., 130, 222

- Leibbestimmtheit 45

- Leiberleben 44

- Leibesgestimmtheit $\quad 43,49$

- Leibeshöhlen 76

- Leibesöffnung 121

- Leibesregungen 47

- Leibesübungen 105

- Leibesverhaltens 48

- Leibesverstimmtheit 49

- Leibphänomenologie 52

- Leibphilosophie 45, 51

- Leibseiender 46

- Leibvergessenheit 50

Leidenschaft $\quad 16,18,22$

Leidensdruck 57, 405

Leidensfähigkeit $298 \mathrm{f}$.

Leidenswelt 294

Leiderfahrung 380,473

Leistung $\quad 40,64,66,73,172,174$ f., 198 , $229 \mathrm{f} ., 297,300 \mathrm{f} ., 306,358,408$

- Leistungsdruck 343

- Leistungsfähigkeit $\quad 4,318,344,346,468$, 472

- Leistungsgedanke 201

- Leistungsgesellschaft 198 
- Leistungskreaturen 308

- Leistungssteigerung 65, 300

Lepra 19, 115, 261

Leviathan $353,355,359$

Liebe $40,75,120,124,298,302 \mathrm{f} ., 377$, 392, 425, 458, 479

Liebesgenuss $120 \mathrm{f}$.

Lieferketten 392

Lifestyle-Medikamente 65

Lobbyismus 308

Logik 23, 72, 319

Luftverschmutzung 269,390

Lunge 170, 250, 264

- Lungenentzündung $435 \mathrm{f}$.

- Lungenmetastasen 302

Lymphgewebe 472

Machbarkeit $32,64 \mathrm{f}$.

Macht $16,55,59,336,357,359,390-392$, 395, 452, $459 \mathrm{f}$.

- Machtakkumulation 24

- Machtbeziehung 17

- Machtposition 19, 459

- Mäeutik 77

Magie 114

Maimonides, Moses 98, 103-105

Malaria $115,142,160,173,250,260,265$

Mangelernährung 481

Mängelwesen 89

Männlichkeit 429

Maschinen-Menschen 20, 48

Massentierhaltung 263, 275, 279

Materialismus 48

Medikalisierung $\quad 59,65,124,426$

Medikament $\quad 40 \mathrm{f} ., 65,115,118,212-214$, 246, 250, 277, 367, 407, 469, 481, 487f., 490, 494-496

Meditation 149

Medizin 1-9, 15-20, 24, 26, 30, 32-36, $39,41,44,48,50,55,57,59,64-67$, 75, 79 f., 89, 97 f., 100, 106, 109 f., $113-$ $116,118,120-122,124-126,128,130$, 133f., 136, 139, 141-143, 146-149, 151, 157 f., 170, 174 f., 177-180, 182 f., 193, 209, 215, 221-226, 228f., 232f., $236-$ 239, 241, 245-249, 251-254, 260, 312f., 316f., 324, 341f., 353-359, 397,
402, 405, 407f., 415f., $436 f ., 439$, $454 \mathrm{f} ., 457,467$

- Medizinalpflanzen 158, 162

- Medizinerausbildung 123

- Medizinethik 67, 312, 346

- Medizinrituale 181

- Medizinsoziologe 4, 318

- Medizintechnik 65, 212

Medizinkonzepte $\quad 26,115,117,122,142,147$, 151, 177, 180, 183, 192, 202, 206 f., 209, 215, 278

Mehrdimensionalität $59,142,410$

Mensch 2-4, 6-10, 16, 22, 25, 27, 31, $33-41,45,47-52,56 f ., 60-62$, $64-66,70-73,75$ f., 78 f., 82 f., 86 , $88 \mathrm{f.,} 91 \mathrm{f} ., 98 \mathrm{f} ., 103-105,113,115,124$, 128, 135 f., 140, 150 f., 153f., 160-162, $166,170,172-174,176,178,180,182 \mathrm{f}$, 193, 196, 201f., $206-208,211 \mathrm{f} ., 225 \mathrm{f}$., 230, 237 f., $240-246,249-253,260$ f., 263f., 268-276, 279-281, 291-296, 299, 302 f., 305f., 308, 313f., 316-319, $325,327,336,340,356,370,383,393$, 405f., 426, 428f., 431, 438, 454, 457f., 460, 462, $468 \mathrm{f}$.

- Menschenaffen 242

- Menschenexperimente 356

- Menschenfeindlichkeit 197, 201

- Menschenrecht 253

- Mensch-Tier-Beziehungen 297

- Mensch-Tier-Ethik 296

Menstruation 99, 213, 250

Mikrobiologie 439

Mikrobiom $276 \mathrm{f}$.

Mikroorganismen 7f., 253, 263, 272, 276

Mismatch 248, $253 \mathrm{f}$.

Mitgefühl 486

Mitleid $83,87,296,458$

Mittelalter $\quad 44,110,120,122,280,296$

Modearzt 222

Modellorganismen 241, $251 \mathrm{f}$.

Monokulturen 175

Morbidität 102,268

mRNA 271

- mRNA-Impfstoffe $270 \mathrm{f}$.

Multilateralismus 393,396 
Mutation 239, 242, 247, 249 f., 315

- Mutationsrate $246 \mathrm{f}$., 263

Muthi $162,165 \mathrm{f}$.

- Muthi-Mischung 162, 165

Mythen 70, $170 \mathrm{f}$.

- Mythologie 140, 200, 211

Nachhaltigkeit $\quad 202,301,411$

- Nachhaltigkeitsforschung 376

- Nachhaltigkeitsziele 5, 157, 254

Nachkriegsmedizin 355

Nahrung 78, 106, 113, 120 f., 125, 151 f., 154, 161, $196 \mathrm{f} ., 199,245,254,292,461,471$

- Nahrungsergänzungsmittel 213

- Nahrungsmittellieferungen 195

- Nahrungsquelle 196, 199

- Nahrungssuche 200

- Nährwerttabellen 470

Nationalsozialismus $\quad 452,454$

Nationalstaaten 5, 103, 138, 157, 254, 389, 391, $394 \mathrm{f}$.

Natur 20, 32, 36-38, 44 f., 65f., 70, 78, $80,111,119,143,170-172,175$ f., 198 f., 201f., 245, 248, 265f., 274, 279, 291f., $294,300,303,306,308,325,353,355$, 411, 428, 451, 460, 479

- Naturgesetze 308

- Naturheilkraft 118

- Naturkatastrophen 111

- Naturlehre 113

- Naturphänomen 279

- Naturphilosophie $222 \mathrm{f}$.

- Naturvölker 193

Naturalismus 61

Naturheilkunde 146, 149

Naturwissenschaft $\quad 34,56$ f., 59 f., 79, 143, 225

Neandertaler $243 \mathrm{f}$.

Nebenwirkung 34, 365, 367, 382, 487, 489, 495

Nerven 152, 223

- Nervenheilkunde 462

- Nervenreiz 23

- Nervensystem 23, 118, 180 f., 331, 474

- Nervenzellen 244, 328

Neuphänomenologe 46

Neuroplastizität 181
Neuroregeneration. Siehe Neuroplastizität

Neurotransmitter 178, 241, 326, 328

Neurowissenschaften 44

Ngubane, Heriette 159

Nietzsche, Friedrich 20, 22-25, 44

Niketas 116, 125

Nikolaos Myrepsos 116, 126

Nikomachische Ethik 37f., 401

Nobelpreis $142,228,436$

Normativität $26,332 \mathrm{f}$.

Notfall 342, 442, 445, 482

- Nofallmedikamente 481

- Notfalldiagnosen 446

- Notfallmedizin 446

- Notfallpläne 384

- Notfallzentren 446

Nutzenmaximierung 407, 409

Nützlichkeit 153, 438

Nutztiere 261, 294, 297, 300, 302f., 306

Obdachlose 431

Obduktion 224

Objektivität 143

Ökobilanz 301

Ökonomie $\quad 23,140,300,408,414$

Ökosysteme 292, 303, 306, 308

Omics-Technologien 237, 239, 268, 277

One-Health $249,253,274,305$

Onkologie 406, 490

Opfertiere 166

Opferzahlen 292

Opiumkriege 138

Oreibasios von Pergamon 115-117, 120

Organ 8, 16, 48, 226, 233, 264, $266 \mathrm{f}$., $276 \mathrm{f} ., 313,319,435,440,447,455$

- Organbeschaffung 358

- Organfunktionen 478

- Organsystem 51, 251

- Organversagen 435, $442 \mathrm{f} ., 479$

Organismus $25,47,57,151,154,247,331$, 333

- Organismusinnenwelt 50

- Organismusmodelle 59

Pädagogik 91, 317

Paläobiologie 248

Paläoökologie 172 
Palliativmedizin 90f., 205

Pandemie $7 \mathrm{f} ., 10,39,80,139,142 \mathrm{f} ., 182$, 238, 249, 253, 261-263, 271f., $274 \mathrm{f}$., 279 f., 292, 341, 380 f., 384f., 387, 393, 395, 397, 435

- Pandemieforschung 279

Papst Franziskus 182

Papua-Neuguinea 195, 205-209, 212-215

Paracelsus 20

Parasiten $264 \mathrm{f}$.

Paternalismus 25

Pathogene. Siehe Krankheitserreger

Pathologie 225, 227, 237, 239, 324, 327, 331

Pathologisierung 59, 452, 459

Pathozentrismus 297

Patient $6 \mathrm{f} ., 9,17,25,31,33 \mathrm{f} ., 36,38-41$, 63 f., 66 f., 72f., 75f., 79, 110, 113f., 119, $124,142,148,158,161,163,165 \mathrm{f}$., $178 f ., 205,210,233,238 f ., 249,252$, 254, 269, 273, 277, 314, 334, 339-343, $347,363-365,368,370,402,404-412$, 414-416, 434f., 439, 441-443, 445, 450, 461f., 467f., 478, 480-484, 488, 491, 495

- Patientenakquise 413

- Patienten-Arzt-Beziehung 408

- Patientenberichte 467-469

- Patienteneinsicht 64

- Patientenerfahrung 410, 447

- Patienteninteresse 447

- Patientennutzen 407, 414

- Patientenperspektive 406

- Patientenpräferenzen 406

- Patientenrechte 491

- Patientensicherheit 344, 440, 447

- Patientenvereinigungen $467 \mathrm{f}$.

- Patientenverhalten 25

- Patientenversorgung 340, 344, 347, 415

- Patientenvertreter 441

- Patientenwohl 415

- Patientenzentrierung 402

- Patientenzufriedenheit 344

- Patientenzugang 342

Paulos von Aigina $115,120-122$

Personal 40, 47, 49, 123, 165, 209, 280, $340-342,347,410,414,446$
- Personalausfälle 341

- Personalausstattung 341f., 345

- Personalbereich 343

- Personalbudget 343

- Personaldecke 341

- Personalentwicklung 346

- Personalkosten 343

- Personalschlüssel 347

- Personalvorgaben 341

Persönlichkeitsmerkmale 193

Person-Sein 206

Pessach 106

Pest $111,115,126,128,138,261,280,291$, 421, 436

- Pest-Delirium 127

- Pestepidemie 261

- Pestleichen 127

- Pestwellen 117

Pfeilgifte 177

Pflanzen 7, 130, 158, 175-178, 183, 199, 211, 224, 240 f., 299, 379

- Pflanzenbestand 162

- Pflanzenkur 176

- Pflanzenmedizin 177, $180 \mathrm{f}$.

- Pflanzenprodukte 158

- Pflanzenschutz 272

Pflege $41,103,135-137,141,154,200$, 333, 339, 341, 346, 372, 426, 489

- Pflegepersonal 473, 494

- Pflege-Personalkosten 343

Pflichtenlehre 356, 359

Phagentherapie 273

Phaidros $77 \mathrm{f}$.

Phänotyp 241, 247, 269, 328

Pharmaindustrie 273, 438

Pharmamarketing 357

Philodemus 17

Philosoph 2f., 16f., 23, 26, 32, 37-39, 43-45, 50f., 55, 71-73, 77f., 81, 87, 98, 104, 110, 116 f., 223, 245, 312, 333

Philosophenarzt 18, 24

Physiologie 223, 227, 237, 251, 294

Physiotherapie 52, $494 \mathrm{f}$.

Pilzinfektionen $275 \mathrm{f}$.

Platon 16-18, 32f., 71-73, 76-79

Plessner, Helmuth 62,460

Plinius 114 
Pocken 173, 182, 261, 421, 436

Polymorphismen 245

Polypragmasie 110

Postmoderne 25, 45, 51

Prädispositionen 151

Pragmatik 59

Prävention 9 f., 39 f., 64, 73, 141, 148, 153, $180,202,226,237,239,248,253,261$, $280,319,326,358,390,423,440$

- Präventionsfähigkeit 10

- Präventionskampagnen 426

- Präventionskörper 420,427-429

- Präventionsmaßnahmen 79, 270, 377, 397

- Präventionsmedizin 238

- Präventionsmodelle 346

- Präventionsmöglichkeiten 292

- Präventionsregimes 427

Praxen 51, 91, 148, 340, 342

Praxisrelevanz 54

Präzisionsmedizin 254

Privatisierung 394

Privatklinik $227 \mathrm{f}$.

Privatpatienten 409

Privatsphäre 372

Prokop von Caesarea 126-128

Proportionenlehre 401

Psyche 80, 143, 202, 278, 487

Psychologie 24, 50, 79, 278, 295, 313, 317

- Psychiater 334, 337

- Psychoanalyse 24, 355, 454

- Psychopathologie 22, 323, 336, 455

- Psychopharmaka 179, 181, 250

- Psychosomatik 143, 462

- Psychotherapeuten 180

- Psychotherapie 50, 52, 58, 90, 181, 331, 450, 452, 455, 462

Pubertät 245

Pulslehre 110, 116, 125

Qualität 3, 47, 109, 113, 148, 151, 158, 180, 232, 301, 344, 347, 404 f., $410-412$, 414, 440-442, 446 f., 495

- Qualitätenmischung 113

- Qualitätseinbußen 408

Quarantäne 138, 305
Rabbiner 97, 102, $105 \mathrm{f}$.

Rassebegriff 303

Rassenhygiene 24

Rassismus 303

Rationalisierung 91, 357

Rationalismus $20 \mathrm{f}$.

Rationalitätskonzepte 26

Raubbau 181, 292, 341

Rauchen 326

Recovery-Ansatz 453

Reduktionismus 49, 91

Regeneration 50, 148, 180, 275

Rehabilitation 317, 336, 342, 458

Religion 18, 62, $90 \mathrm{f}$., 97-99, $105 \mathrm{f}$.

Rente $181,368,384,454,458,476,482$, 490

Reproduktion 213, 252, 355

Resilienz 139f., 151f., 316f., 346, 381, 453

- Resilienzförderung 319

Resistenzen 238, 263, 272f., 276, 306

- Resistenzgen 276

Resonanzkörper 49

Resonanz-Metaphern 50

Resonanzwirkung 51

Ressourcenverbrauch 301, 306

Rettungsdienste 340

- Rettungshubschrauber 494

- Rettungsleitstellen 446

- Rettungssanitäter 342

Risiko 31, 173, 243, 262, 268, 275, 292 , $313-315,317-319,344,355,373,376-$ 385, 391, 407, 423, 425 f., 442, 444, $470 \mathrm{f}$.

- Risikobereitschaft 10

- Risikofaktorenmodell 316

- Risikogruppe 420, 423, 425, 491

- Risikokommunikation 381

- Risikopatienten 273

- Risikorat 377, 383

- Risikoübernahme 384

- Risikoverhalten 420, 423

- Risikoverursacher 382

- Risikowahrnehmung 280

Ritual 85, 163-166, 198, 206, 211, 215

Rollenbilder 171, 193

Romantik 297, 299

Roth, Walter Edmund 192, 200 
Routinedaten 364-370

Routineuntersuchung 238,470

Rückenschmerzen 163-165

Salutogenese $312,317,319$

SARS 142, 274, 305, 388, $394 \mathrm{f} ., 398,425$, 431

SARS-CoV-2. Siehe COVID-19

Schadenszauber 208-210, 212f., 215

Schattenseele $207 \mathrm{f}$.

Scheinhilfsbereitschaft 52

Schlaf 35, $86 \mathrm{f} ., 104 \mathrm{f.,} \mathrm{113,} \mathrm{136,} 154$

- Schlafkrankheit 160, 260

- Schlafmangel 344

- Schlafmittel 228

- Schlafstörungen 57

Schleim 16, 113, 150, 214

Schmerzen 46 f., 50, 77, 90 f., 124, 214, 226, 248, 250, 294, 296f., 314, 478, 480, $482 \mathrm{f} ., 494$

Schmitz, Hermann 16, 43-47, 49-52

Schopenhauer, Arthur 50

Schulmedizin 105, 148

Schulunterricht 253, 494

Schutzheiligenverehrung 110 f., 113

Schwangerschaft 213f., 271, 366

Schweigepflicht 64

Schweine 212, 261, 294, $300 \mathrm{f}$.

- Schweinegrippe 291, 435

- Schweinepest 300

Schwerbehinderung 473

- Schwerbehindertenausweis 490

- Schwerbehindertenrecht 472

Seele $15-18,24,32,37 \mathrm{f} ., 44,48-50,71$, $78 \mathrm{f} ., 84,87-90,102-104,106,152 \mathrm{f}$.

- Seelenarzt 17

- Seelenfunktionen 126

- Seelenkrankheiten 17

- Seelenleiden 120

- Seelenruhe 44

- Seelentheorie 126

Seelsorge 38,90

Selbstbewusstsein 196, 201, 296

Selbstdarstellung 298

Selbsterfahrung $332 \mathrm{f}$.

Selbsterkenntnis 44, $332 \mathrm{f}$.

Selbstermächtigung 16
Selbsterzählung 318

Selbstführung 426

Selbstheilung 25

Selbsthilfe 54, 468, 471, 477, 479f., 484, 491

Selbstkontrolle 37

Selbstmanipulation 56

Selbstnormierung 404

Selbstregulierung 23

Selbstschädigung 426

Selbstverantwortung 409

Selbstvergessenheit 313

Selbstverständnis 176, 195

Selbstvertrauen $\quad 454,474$

Selbstwertgefühl $\quad 469,480$

Selbstwirksamkeit $\quad 454$

Sensibilität 378

Sepsis 121, $434 \mathrm{f} ., 440-447$

- Sepsis-Krankenhaussterblichkeit 443

- Sepsisplan 440, 446

- Sepsisresolution 440

Sequenzierung 238

Sesshaftigkeit 70, 244

Settingansatz $79 \mathrm{f}$.

Seuche $126-128,158,261,291,420 \mathrm{f}$. 425, $436 \mathrm{f}$.

- Seuchenausbrüche 253, $262 \mathrm{f}$.

- Seuchenbekämpfung 19, 138

- Seuchengeschichte 115

- Seuchenvermeidung 436

Sexualität $\quad 35,89,154$

Sexualkontakte 457

Sexualmediziner $\quad 425$

Sexualmoral 99

Sexualpraktiken 426

Signalkaskade 268

Sinne 153

- Sinnenlust 121

- Sinnerleben 344

- Sinneseindrücke 151, 245

- Sinnesorgane $152 \mathrm{f}$.

- Sinnesstörungen 304

Sokrates 71f., 74, 76-78

Sontag, Susan 26

Sozialbeziehungen 208, 215

Sozialdarwinismus 27, 252

Sozialfürsorge 102 
Sozialgesetzbuch 415

Sozialhilfe 472,479

Sozialhygieniker 102

Sozialisation 193

Sozialkonstruktivismus $332 \mathrm{f}$.

Sozialmedizin 80

Sozialpartnerersatz 298, 305

Sozialpsychologie 333

Sozialreformen 458

Sozialstruktur 176, $207 \mathrm{f}$.

Sozialverhalten 202, 278

Sozialversicherung $\quad 365,369$ f., 372

Spiritualität $90 \mathrm{f}$., 252

Sport 35, 193f., 198-202, 486

- Sportlichkeit 75

- Sportmedizin 65

- Sportpädagoge 192, 201

- Sportprogramm 192, 197

- Sportunterricht 193, 201

Stammeskultur 193

Stammzellen 252

Stammzelltransplantation $\quad 473$

Sterberegister 365,369

Sterblichkeit $\quad 63,65,127,135,231,438$

Sterblichkeitsrate 127, 377, 445

Sterblichkeitsrisiko 441

Stigmatisierung $61,213,319,368$

Stimulanzien 24

Stoffwechsel 8, 23, 113, 151f., 241, 248, 277, 280

- Stoffwechselerkrankungen 249, 300

- Stoffwechselprodukte 277

Stress 36f., 295, 305, 339f., 342, 344, 384, 470, 476

- Stressbewältigung 346

Subjektivität 143

- Subjektivität/Objektivität 26

Substanzmissbrauch 180

Suchterkrankung 344f., 456, 458

Symptom-Behandlung 75

Systemrelevanz 272

Tacitus, Publius Cornelius 99

Talmud 97, 104-106

Tanzen 9, 476

Technikfolgenabschätzung 67

Teilhabe 15, 202, 452, 461, 483
Temperaturanstieg 275

Tertullian 111

Theodora 117, 128

Theodoros Prodromos 123

Theodosius 86, 115

Theophilos 123

Theophrast 110

Therapie $15-17,24,58,64,67,71 f ., 78$, $86 \mathrm{f} ., 113,148,151,153,158,181,183$, 207, 210f., 215, 233, 237, 252f., 269, 272, 274, 276, 299, 317f., 334, 365, $405-407,439,441,443,468,472-$ 474, 487, 490f., $494 \mathrm{f}$.

- Therapieabschluss 318

- Therapieangebote 460

- Therapieansatz 273

- Therapiemöglichkeit 253f., 273, 429, 469, 496

- Therapienotstand 306

Thora 98, 103, 105

Tiere 7, 130, 175f., 178, 181, 199, 211, 224, 240, 251, 253, 261, 274f., 279, 291-301, 303, 306, 308, 379

- Tierexperimente 252

- Tiergerechtheit $294 \mathrm{f}$., $302 \mathrm{f}$.

- Tiergesundheit 293-296, 300 f., $305 \mathrm{f}$.

- Tierleid 295f., 299

- Tierliebe $297 \mathrm{f} ., 305$

- Tiermedizin $299 \mathrm{f}$.

- Tierreservoir 292

- Tierschutz 294, $298 \mathrm{f}$., $307 \mathrm{f}$.

- Tierversuch 299

- Tierwohl 294-296, 298f., 301, $306 \mathrm{f}$.

Timaios 77-79

Todesangst 111

Todesfälle 161, 208, 272, 340, 435 f., 440, 442, 448

Todesopfer 199

Todesrate 126

Todesursachen 140

Todeswille 50

Totengeist $207 \mathrm{f}$.

Totenseele 207

Totgeburt $214 \mathrm{f}$.

Transplantationsmedizin $\quad 357$

Transzendenz 134

Trauer 57f., 200, 460 
Traumata 141

Trinkwasser 200, 390, 436

Tuberkulose 214, 227, 261, 265, 268, 292, 314, 436, 455

Tumorerkrankung. Siehe Krebserkrankung

Tutu, Desmond 166

Überarbeitung 1, 303, 307

Übergewicht 277, 326, 470

Überlebensrate $442 \mathrm{f}$.

Übertragung $19,22-24,110,252$

Überwachung $61,367,372 \mathrm{f}$.

Überzüchtung 272

Ubuntu 166

Umwelt $\quad 7-9,31,33 \mathrm{f} ., 50,59,72 \mathrm{f} ., 75 \mathrm{f}$., $80,98,157,162,166 \mathrm{f.}, 174,176,183$, 199, 201, 212, 215, 244, 247, 253, 272, 294, 299, 301, 306, 331f., 334, 366, $377,382 \mathrm{f}$.

- Umweltbedingungen 243, 246-248, 252, 453

- Umwelteinflüsse $\quad 49,248,305,367$

- Umweltressourcen 301

- Umweltstandards 272

- Umweltveränderungen $377,382 \mathrm{f}$.

- Umweltzerstörung 56,183

Universalmedium 136

Universität $\quad 1,30,43,54,70,82,90$ f., 109, 133, 141, 146, 158, 205, 221-223, 225, 227 f., 236, 291, 355, 363, 401, 420 f., 467

- Universitätsklinik 227, 229, 429

- Universitätsmedizin $\quad 233,236,241,353$, 434, 450, 467

Unsterblichkeit $133 \mathrm{f}$.

Urbanisierung 253, 263, 292

Ureinwohner 192-201, 203

Urteilsfindung 377

Urteilsvermögen 38

Valentinian I. 116

Verbraucherschutz 308

Verdauung 105,151-153, 295

- Verdauungsstörungen 129

- Verdauungstrakt 178

Vererbung 138

- Vererbungsgeschehen
- Vererbungslehre 252

- Vererbungsvorstellungen 125

Vergesellschaftung 394

Vergütungssystem 412

Verhalten $7 f ., 23,36,39 f ., 60,78,120,151$, 154, 199, 201, 208, 233, 253, 278, $294 \mathrm{f.,}$ 297, 301, 316, 329, 332, $425-427,450$, 452f., 456, 459

- Verhaltensänderung $\quad 89,278,425,456$

- Verhaltensauffälligkeiten 455

- Verhaltenskodizes 211

- Verhaltensmuster 295, 452, 459

- Verhaltensnormen 61

- Verhaltensrepertoire 295

- Verhaltensstörungen 301

- Verhaltenstendenzen 460

- Verhaltenstherapie 40, 454

- Verhaltensverarmungen 295

- Verhaltensvielfalt 462

Verhältnisprävention 316

Verhexung 111

Verliebtsein 120

Vernunft $15-18,20-25,37,78$

- Vernunftbegriffe 20, 24

- Vernunftkonstruktion 15

- Vernunftkonzepte 15

- Vernunftkritik $21 \mathrm{f}$.

Verschwörungstheorien 280

Versicherungen 367,487

- Versicherungsmarkt 368

- Versicherungsmodelle 382

- Versicherungsobligatorium 408

- Versicherungsprämien 382

- Versicherungssystem 408

Versorgung $4,6,9,36,39,122,139,157 \mathrm{f}$., $160,177,193,214,226,228,232,237 \mathrm{f} .$, 277, 340 f., 346f., 358, 368, 401-416, 480, 482

- Versorgungsdienste 124

- Versorgungskapazitäten 458

- Versorgungslücken 491

- Versorgungsqualität 344f., 347, 410, 414

- Versorgungssystem 158, 411, 491

- Versorgungsszenario 340

Versuchstiere 294f., 297-299, 306

- Versuchstierleid 299, 307

Verteilungsgerechtigkeit $\quad 401,410$ 
Veterinärämter 307

Veterinärmedizin 271, 291

Vielstimmigkeit 142

Viersäftelehre 19

Vier-Säulen-Konzept 410

Virchow, Rudolf 1f., 99, 221, 224-233, 239, 269, 359, $436 \mathrm{f}$.

Virologie 421

Virulenz 126

Virus $10,242,270,292,305,377,382$, 398, 491

Vitalität 141, 152, 378

Vitaminmangel 481

Vogelgrippe $253,291,435$

Völkerbund 389

von Weizsäcker, Viktor $50 \mathrm{f}$.

Vorsokratiker 113

Vorsorge 238, 358f., 377-385

- Vorsorgeuntersuchung 31

Wahrnehmung $16,88,149,162,202,303$

- Wahrnehmungsverzerrungen 308

Wahrscheinlichkeit $\quad 31,35,376,378 \mathrm{f} ., 383$, 447

- Wahrscheinlichkeitstheorie 380

- Wahrscheinlichkeitsverteilung $376 \mathrm{f}$.

Waldinseln 172, 175

Waldkindergärten 202

Waldpädagogik 202

Waschungen 99, 207, 215

Wasser $73,75,127,136,159,171,199-201$, 273

- Wasserqualität 75

- Wasserquellen 195

Wassmann, Jürg 206

Weißkittelsyndrom 488

Wellness 35

- Wellness-Ideologie 52

- Wellness-Industrie 52

Weltärztebund 339, 344, 404

Weltgesundheitsorganisation 3f., 80, 90, 142, 147, 157 f., 165 f., 179 f., 262 f., 279f., 312, 317, 340, 387-398, 406, 409 f., 434 f., 438, 440-442, 450 f., 454, 462

- WHO-Begriff 454

- WHO-Definition 166, 451
- WHO-Gesundheitsdefinition 65

- WHO-Verfassung 390

Weltgesundheitsversammlung 390 f., 395

Weltklima 171

Wertschätzung $97,135,193$

Wertung 381, 454, 456, 462

Werturteil 324, 328, 380, 408

Wertvorstellungen $323 \mathrm{f} ., 356,410$

Wettbewerb 198, 238, 357, 476

- Wettbewerbsverzerrung 65

Wildnis $171,182,275,295$

Wildtiere 292f., 295-297, 300

Wildtiergesundheit $305 \mathrm{f}$., 308

Willenskraft 458

Wirklichkeitskompetenz 59

Wirkmechanismen 181

Wirksamkeit 17, 25, 139f., 143, 149, 158, 227, 230, 326, 367, 390, 404, 406

Wirkstoff $178,181,250,272$

Wirt 292

Wirtschaftlichkeit $\quad 301,405,409,414$

- Wirtschaftlichkeitsdruck 346

Wissenschaft $3-5,9,20,23,26,30,32$, $55-59,65,71,82,89-92,138 \mathrm{f} ., 142 \mathrm{f} .$, 147, 149 f., 153f., 157, 170, 221, 223, 226, 228, 236 f., 248, 252f., 260, 263, 277, 306, 355, 380 f., 383f., 389, 391, 408, 421, 437, 448, 455, 467, 490

- Wissenschaftsgeschichte 109, 356

- Wissenschaftskommunikation 280

- Wissenschaftspublikationen 149

- Wissenschaftssprache 244

Wissenskorpus 167

Wissensübertragung 159

Wissenszuwachs 178,183

Wittgenstein, Ludwig $\quad 25,322,337$

Wohlfahrt 358

- Wohlfahrtspflege 346

Wohlgefühl 2, 9, 312

Wohlsein 8, 140

Work-Life-Balance 341

World Health Organization. Siehe Weltgesundheitsorganisation

Wunder 44, 84 f., 89 f., 92, 119, 337, 429

- Wundergeschichten 85

- Wunderheiler 162 
- Wunderkuren 119

Würdigkeit 401

X-Chromosom 250

Xenodochien 122, 124

Xenon 123

Yogatherapie 146

Yupno 205-207, 209, 211f., 215

Zähne 137, 397

- Zahnärzte 440

- Zahnfleischentzündungen 278

Zaubermittel 119

Zaubersprüche 114

Zelltheorie 233

- Zellbiologie 226, 233

- Zellschäden 227

- Zellteilung 241
- Zelltherapien 269

- Zelltod 266

- Zellularpathologie 227 f., 230

- Zellveränderung 315

Zivilgesellschaft $\quad 358,393$

Zivilisationskrankheiten $\quad 8,70,438$

Zoonosen 261, 274, 291f., 303, 305

Zuchtfolgen 302

Zucker 8, 161, 196, 470

Zulassungsverfahren 271

Zusatzstoffe 103, 481

Zuschüsse 382

Zwangseinweisung 336

Zweckmäßigkeit 296

Zytokine 241, 267, 435

- Zytokin-Familie 267

- Zytokin-Sturm 435

Zytologie 233 
